\title{
Mission d'étude du programme ANR « Géologie et architecture à Délos » - 2020
}

Rapport 2020

Jean-Charles Moretti, Pavlos Karvonis et Tommy Vettor

\section{(2) OpenEdition}

Journals

Édition électronique

URL : http://journals.openedition.org/baefe/1748

DOI : $10.4000 /$ baefe. 1748

ISSN : 2732-687X

Éditeur

ResEFE

Référence électronique

Jean-Charles Moretti, Pavlos Karvonis et Tommy Vettor, « Mission d'étude du programme ANR « Géologie et architecture à Délos » - 2020 » [notice archéologique], Bulletin archéologique des Écoles françaises à l'étranger [En ligne], Grèce, mis en ligne le 01 novembre 2020, consulté le 22 mars 2021. URL : http://journals.openedition.org/baefe/1748 ; DOI : https://doi.org/10.4000/baefe.1748

Ce document a été généré automatiquement le 22 mars 2021.

\section{cc) $(1) \odot$}

Le Bulletin archéologique des Écoles françaises à l'étranger est mise à disposition selon les termes de la Licence Creative Commons Attribution - Pas d'Utilisation Commerciale - Pas de Modification 4.0 International. 


\section{Mission d'étude du programme ANR « Géologie et architecture à Délos » - 2020}

Rapport 2020

Jean-Charles Moretti, Pavlos Karvonis et Tommy Vettor

\section{NOTE DE L'AUTEUR}

En 2020, la crise sanitaire a conduit à réduire les durées, les effectifs et les objectifs des campagnes de terrain du programme « Géologie et architecture à Délos ", qui a débuté en 2019. La mission prévue à Délos en mai-juin a été annulée et celle d'août-septembre n'a rassemblé que trois chercheurs durant trois semaines (24 août-11 septembre).

Autorité nationale présente : Éphorie des Cyclades

Numéro de mission : C56

Établissements porteurs de l'opération : EFA

Données scientifiques produites :

Carnet Hypothèses

Chroniques de l'EfA :

Géologie et architecture - 2019

\section{Les marbres}

Tommy Vettor

1 Dans le cadre du travail conduit sur les marbres architecturaux, 45 prélèvements ont été réalisés sur des blocs du temple de Létô, du Monument aux hexagones, du temple d'Héra, du Temple des Déliens, du Temple des Athéniens, du Prytanée, de 
l'Ekklésiastérion, du théâtre, de la Salle hypostyle et sur une colonne de la Rue du péribole. Ces prélèvements feront l'objet de trois types d'analyses: minéralogiques, géochimiques et isotopiques. Dès à présent ils ont fait l'objet d'analyses in-situ non destructives utilisant un microscope de terrain. Des photographies ont été prises pour chaque bloc à l'aide du microscope, qui permettront d'étudier la taille des grains de calcite de chaque marbre prélevé, un paramètre clef dans la détermination des provenances de marbres.

2 Afin de poursuivre ce mode d'approche, une liste de prélèvements de marbre sur d'autres éléments architecturaux a été élaborée et sera proposée pour la campagne de mai 2021. Au cours de la planification de ces prélèvements, dans la cour de l'Agora des Italiens, sept blocs du stylobate nord du péristyle ont été reconnus comme étant en marbre de Livadia (fig. 1), une provenance qui reste à confirmer par des analyses tant micro-destructives que non destructives. Ils alternent dans l'assise avec des blocs de scorie andésitique suivant une combinaison attestée dans certaines constructions de l'ancienne Théra.

Fig. 1. Agora des Italiens, stylobate du portique nord, extrémité est, du sud-ouest : un bloc probablement en marbre de Livadia entre deux blocs de scorie andésitique.

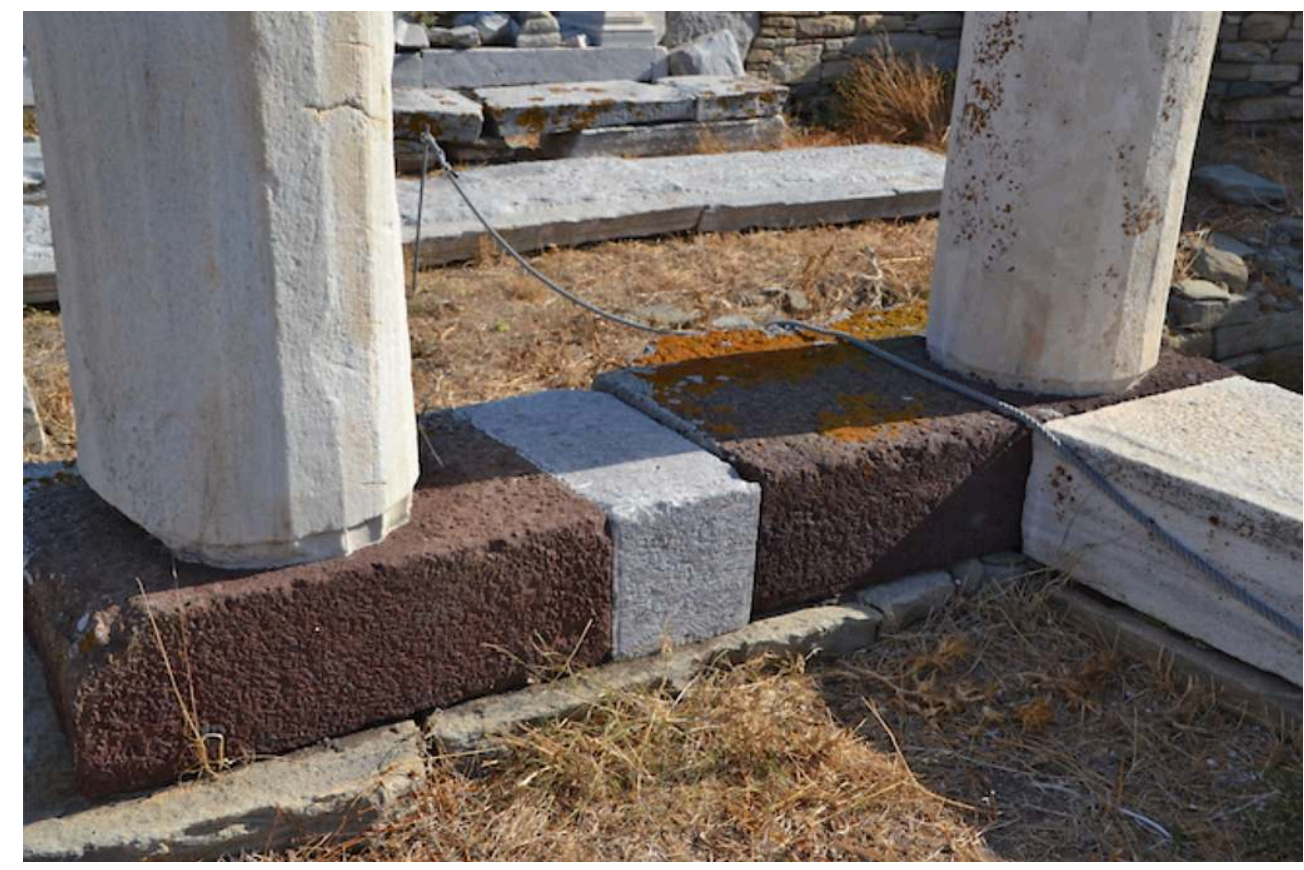

GAD / EFA, J.-Ch. Moretti.

3 La carrière de marbre C25 (sud-est du Cynthe) a été à nouveau échantillonnée méthodiquement à différents niveaux, plus ou moins éloignés du contact avec le granite. Cela permettra d'évaluer les variations isotopiques au sein de la carrière. Les différents niveaux de la carrière de marbre C32 (à l'ouest de la terrasse en gamma) ont été échantillonnés et feront l'objet d'analyses micro-destructives géochimiques et isotopiques.

4 L'étude de la géométrie des lentilles de marbre à Délos s'est poursuivie avec une cartographie précisée au niveau de la carrière centrale et largement complétée au niveau de la colline de Glastropi où des niveaux très grossiers (similaires à ceux de la carrière (25) ont été repérés et prélevés. 


\section{Les carrières de gneiss et de granite du nord de l'île}

Pavlos Karvonis et Jean-Charles Moretti

5 La description des carrières identifiées dans l'île a été poursuivie. Elle a commencé par la vingtaine de carrières du nord de l'île (fig. 2) réparties entre la colline de Gamila et les deux presqu'îles de Patinioti, à l'est, et du cap Morou, à l'ouest. Du granite et du gneiss rubané micacé, de couleur vert ou gris sombre principalement, en ont été extraits.

Fig. 2. Les carrières du nord de Délos.

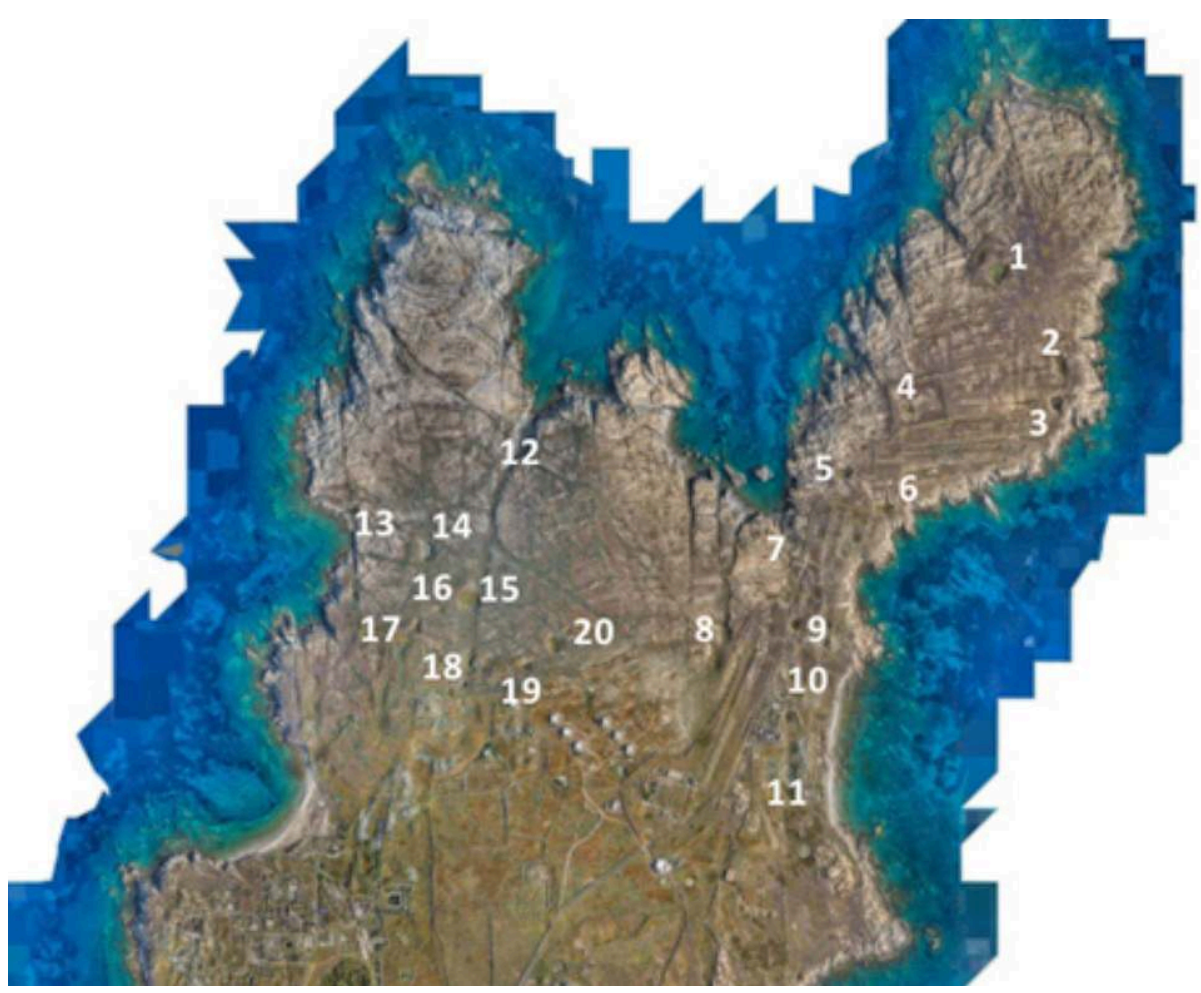

Numérotation sur l'orthophotographie réalisé par Iconem.

Iconem.

6 Le granite a été extrait en fosse. Son mode de fracturation n'a pu être déterminé, faute de vestiges significatifs. Les carrières de granite du Quartier du stade (C8, C9, C10, C11) semblent avoir été ouvertes pour les constructions du quartier : le stade, le xyste et le gymnase, qui sont presque entièrement construits dans cette roche, mais aussi les maisons à l'est, au nord et au sud du stade. Les carrières ont été incluses dans la trame du quartier quand il s'est développé. Elles n'ont pas été remblayées, et une (C10) voire deux (C11) d'entre elles ont été bordées de murs.

7 Le gneiss était suffisamment fracturé pour qu'il fût possible d'extraire des moellons simplement en utilisant un levier métallique, sans avoir recours à des saignées, des emboîtures ou des encoignures. La grande carrière C13 située en bord de mer sur la côte occidentale fait exception à plus d'un titre. Le gneiss y affleurait en sorte qu'il n'a pas été nécessaire pour l'exploiter de creuser sous le niveau du le sol environnant. Il était peu fracturé ce qui a permis d'extraire des blocs pouvant atteindre $3 \mathrm{~m}$ de 
longueur. Bien que la situation de $\mathrm{C} 13$ en bord de mer soit peu favorable à la conservation des traces de taille, nous y avons identifié quelques vestiges d'emboîtures. Cette carrière est aussi la seule qui est associée à deux petites criques qui ont pu recevoir des embarcations pour le transport maritime des blocs. Dans les autres, l'extraction s'est faite en fosse. Après qu'une couche plus ou moins importante de découverte ait été retirée, les moellons extraits ont été transportés par voie terrestre jusqu'à leur lieu de mise en œuvre.

8 Parmi ces carrières de gneiss à extraction en fosse, celles de Patinioti se distinguent par leur environnement construit composé de murs de clôture ou de terrasse et, pour certaines $(\mathrm{C} 1, \mathrm{C} 2, \mathrm{C} 3, \mathrm{C} 6)$, par les rampes simples ou doubles qui ont été creusées entre le sol de la carrière et celui du terrain environnant (fig. 3). Ce dernier dispositif est aussi attesté pour deux carrières du cap Morou $(\mathrm{C} 17, \mathrm{C} 18)$.

Fig. 3. La carrière $\mathrm{C} 3$, du nord-ouest.

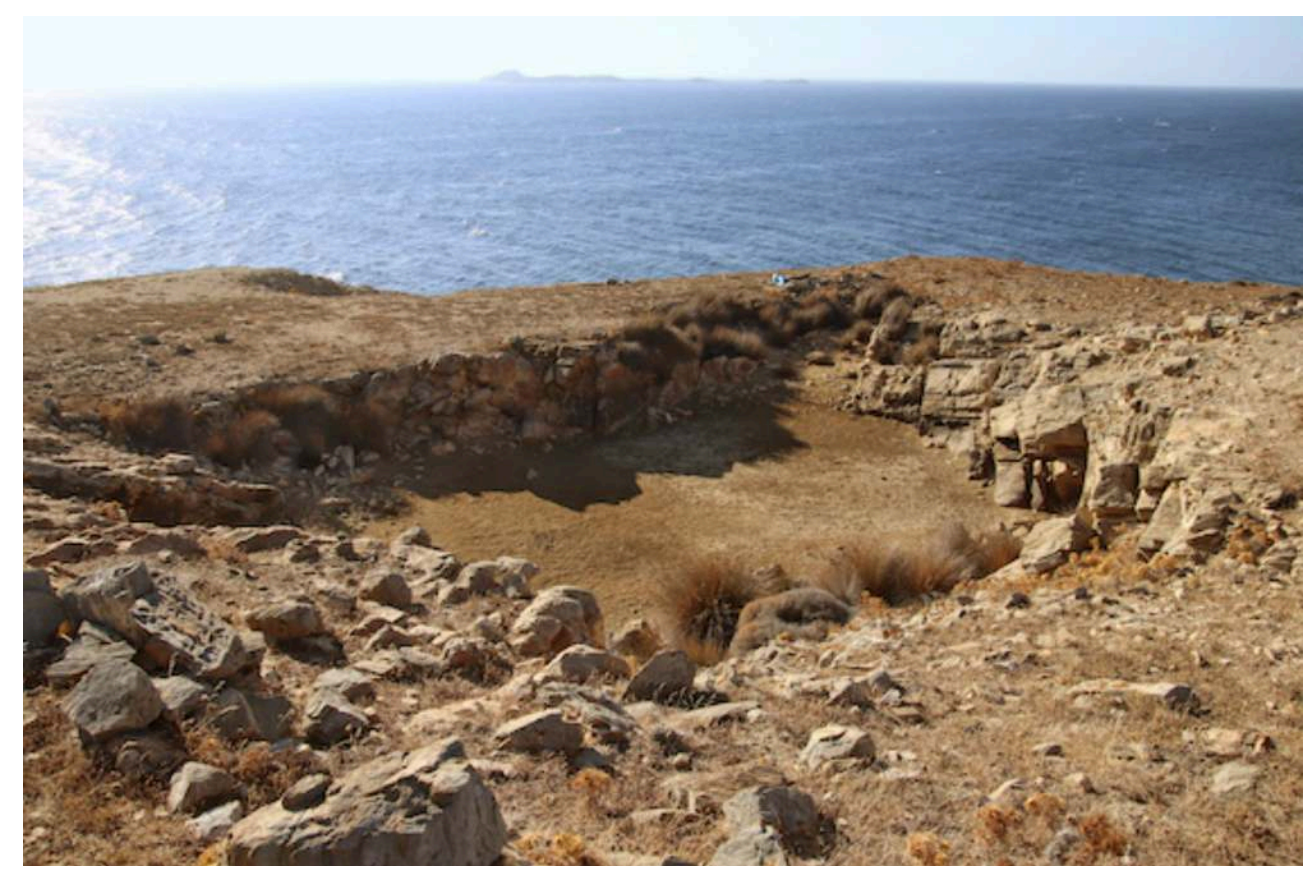

GAD / EFA, P. Karvonis.

9 Le plan des carrières au moment de leur abandon est pour beaucoup plus ou moins semi-circulaire avec un front rectiligne en granite (C3, C9, C10, C14, C16, C17, C18, C19). Il est plus rarement circulaire $(C 1, C 15, C 20)$ ou rectangulaire $(C 2, C 5, C 6)$. Dans deux cas la carrière a élargi un talweg $(\mathrm{C} 8, \mathrm{C} 12)$; dans un autre elle s'est développée sur une longueur nord-sud de plus de $100 \mathrm{~m}$ (C11) ; et dans un dernier elle s'est implantée en bordure d'une faille nord-sud située à la base de la côte occidentale de la presqu'île de Patinioti (C7).

Les gneiss micacés de couleur verte ou grise extraits dans les carrières du nord de l'île sont très présents dans le Quartier du stade : dans les cloisons intérieures du magasin $\varepsilon$ de la partie ouest de la Maison I E, dans les constructions entre les Maisons I A et I B (boutiques $\alpha-\delta$ ), et dans les couches de destruction visibles des maisons non fouillées. Ils le sont aussi dans certaines constructions d'époque athénienne situées à la périphérie du lac : l'Agora des Italiens, le Sanctuaire du bastion à l'exception du temple, 
les constructions au nord du Sanctuaire du bastion, les magasins à l'ouest de la Palestre du lac, les latrines de la Palestre du lac et les magasins adossés à son flanc ouest, ainsi que quelques murs intérieurs de l'édifice commercial au nord du lac et les constructions au nord de la Terrasse des lions. Dans certaines constructions du Quartier $\mathrm{du}$ lac, et non des moindres, le gneiss de Délos est cependant totalement absent. C'est le gneiss à mica blanc de Rhénée, taillé en blocs rectangulaires, qui a été mis en œuvre dans les élévations de la Palestre de granit, de l'Établissement des Poséidoniastes de Bérytos, du Monument de granit, des Maisons du lac, de Skardana, du Diadumène, des tritons et des comédiens et dans presque toutes celles de l'îlot des bijoux.

11 Dans ces maisons des Quartiers du stade et du lac, les gneiss du nord de lî̂le apparaissent dans des appareils de moellons très frustes, sans assises régulières (fig. 4). Le plus souvent ils se trouvent dans des parois élevées lors de réfections ou d'agrandissements (Agora des Italiens, Palestre du lac). Les gneiss sont le plus souvent soit très majoritaires, soit associés à du granite local dans des maçonneries où la distinction des natures de roches ne semble avoir joué aucun rôle. Dans ces maçonneries le choix paraît avoir été de retenir des matériaux locaux sans distinction des différents types de roches.

Fig. 4. Magasin au sud de l'Agora des Italiens, mur entre deux boutiques, parement en gneiss de Délos.

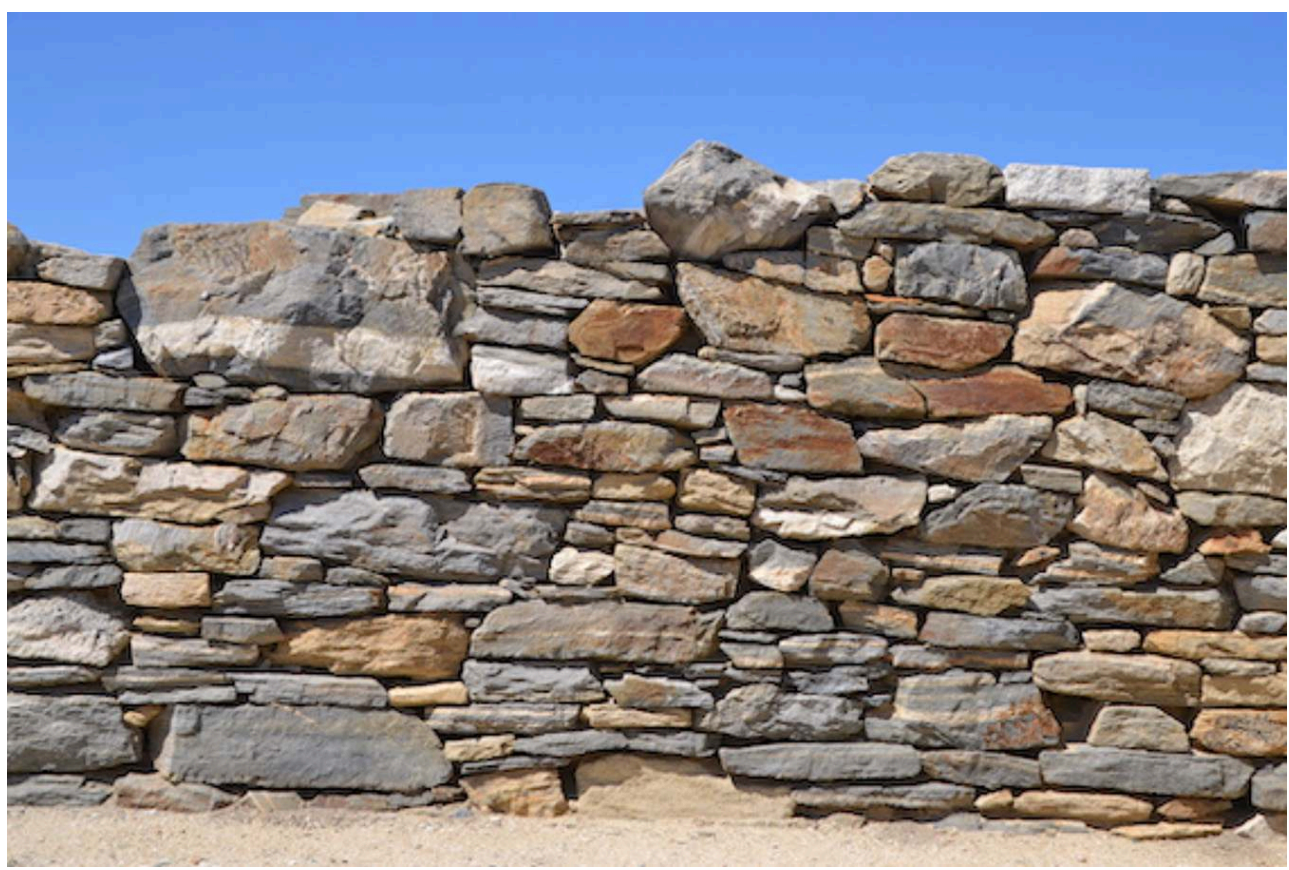

GAD / EFA, J.-Ch. Moretti.

Plus on s'éloigne du lac vers le sud, moins le gneiss du nord de l'île est présent dans l'architecture domestique. Il l'est majoritairement dans la fondation de la colonnade qui borde à l'est la Rue du péribole, mais au-delà on ne trouve que quelques blocs disséminés dans les murs de quelques maisons, comme la Maison de Kerdon. Dans le Quartier du théâtre, il n'apparaît dans des proportions significatives que dans deux murs de l'Insula IV et dans le mur sud de la Maison du trident.

13 Ce gneiss n'est pas absent dans les sanctuaires d'Apollon et d'Artémis. Il apparaît sporadiquement dans certains vestiges mycéniens, comme ceux qui sont pris dans le 
Trésor 5, dans des fondations de monuments archaïques ou classiques (Monuments aux hexagones, autel de l'Artémision, Porinos naos, Trésor 5, GD 48), dans de rares élévations (parement, intérieur surtout, du temple archaïque d'Artémis) et dans des fondations de bases d'époque hellénistique.

Pour autant que nos identifications à vue sont justes, les gneiss micacés gris et vert sombre de Délos apparaissent donc avoir été mis en œuvre dès l'époque mycénienne sous forme de moellons. Aux époques archaïque et classique, ils sont aussi connus sous forme de moellons dans des élévations, mais plus souvent sous celle de grandes plaques de formes variées en fondation. Après une éclipse à l'époque de l'Indépendance, ils réapparaissent à l'époque athénienne à la fois en fondation et en élévation sous forme de moellons bruts de carrière.

\section{INDEX}

Thèmes : EFA

Année de l'opération : 2020

sujets https://ark.frantiq.fr/ark:/26678/pcrts8SiTTY3Ka, https://ark.frantiq.fr/ark:/26678/

pcrtXk6sdvTjnE, https://ark.frantiq.fr/ark:/26678/pcrtxzTYzVBKas, https://ark.frantiq.fr/ark:/

26678/pcrtSkipOsBGML

lieux https://ark.frantiq.fr/ark:/26678/pcrtXvsaqTQa4J, https://ark.frantiq.fr/ark:/26678/

pcrtKgel43Esf

chronologie https://ark.frantiq.fr/ark:/26678/pcrtNzYn31IIAZ

\section{AUTEURS}

\section{JEAN-CHARLES MORETTI}

Institut de recherche sur l'architecture antique, CNRS, Maison de l'Orient et de la Méditerranée

\section{PAVLOS KARVONIS}

Académie d'Athènes

\section{TOMMY VETTOR}

Muséum National d'Histoire Naturelle, UMR 7590 « Institut de minéralogie, de physique des matériaux et de cosmochimie» 